\title{
具有聚集诱导发光效应的聚炔材料的合成及光学性能研究
}

\author{
韩婷 $\dagger, a, b \quad$ 詹嘉慧 ${ }^{\dagger}, a, b \quad$ 林荣业*,a,b唐本忠*,a,b \\ ( ${ }^{a}$ 香港科技大学化学系 国家人体组织功能重建工程技术研究中心香港分中心 香港九龙清水湾) \\ ( ${ }^{b}$ 香港科技大学深圳研究院 深圳 518057)
}

\begin{abstract}
摘要 合成了三种含有四苯基乙烯结构的双炔烃. 这些双炔烃单体通过在 $\mathrm{CuCl}$ 催化下在邻二氯苯中进行的均聚反应 可以高产率地生成线型聚炔. 所得聚合物均可很好地溶解于常见有机溶剂中, 并具有很高的热稳定性. 这类聚炔材料 在溶液态时几乎不发光，但在聚集态或者固态下可高度发光，表现出典型的聚集诱导发光性质. 同时，这些聚合物具 有高透光性，可以允许整个可见光区的光透过. 其薄膜在 $400 \sim 1700 \mathrm{~nm}$ 宽波长范围内表现出高折射率 $(n=1.7787 \sim$ $1.6543)$ 和低色差 $\left(D^{\prime}=0.0003\right)$. 紫外光照射可诱使聚合物薄膜发生交联过程, 从而调控其折射率数值, 并可生成高分辨 率的苂光图案.
\end{abstract}

关键词 线型聚炔; 聚集诱导发光; 四苯基乙烯; 折射率

\section{Polyynes with Aggregation-Induced Emission Characteristics: Synthesis and Their Photonic Properties}

\author{
Han, Ting ${ }^{\dagger, a, b}$ \\ Cathy K. W. Jim ${ }^{\dagger}, a, b$ \\ Jacky W. Y. Lam*,a,b \\ Tang, Ben Zhong*,a,b
}

$\left({ }^{a}\right.$ Department of Chemistry, Hong Kong Branch of Chinese National Engineering Research Center for Tissue Restoration and Reconstruction, Institute for Advanced Study, Institute of Molecular Functional Materials, Division of Biomedical Engineering, Division of Life Science and State Key Laboratory of Molecular Neuroscience, The Hong Kong University of Science \& Technology, Clear Water Bay, Kowloon, Hong Kong, China)

$\left({ }^{b}\right.$ HKUST-Shenzhen Research Institute, No. 9 Yuexing 1st RD, South Area, Hi-tech Park, Nanshan, Shenzhen 518057, China)

\begin{abstract}
Tetraphenylethene-containing diacetylenes are synthesized and their homopolymerizations are catalyzed by $\mathrm{CuCl}$ in $o$-dichlorobenzene, furnishing linear polyynes in high isolated yields. All polymers possess good solubility in common organic solvents and enjoy high thermal stability. Whereas they are practically non-emissive when molecularly dissolved in good solvents, they become highly emissive when aggregated as nanoparticle suspensions in poor solvents or fabricated into thin films in the solid state, demonstrating a novel phenomenon of aggregation-induced emission. The polymers are highly transparent, allowing almost all light in the entire visible spectral region to transmit through. Thin films of the polymers show high refractive indices $(n=1.7787 \sim 1.6543)$ in the wavelength region of $400 \sim 1700 \mathrm{~nm}$ and very low chromatic aberrations $\left(D^{\prime}=0.0003\right)$. Their $n$ values can be modulated and their thin films can be crosslinked by UV irradiation, generating fluorescent patterns with high resolutions.
\end{abstract}

Keywords linear polyynes; aggregation-induced emission; tetraphenylethene; refractive index

\section{1 引言}

如何简单高效地制备出具有新型结构和独特性质 的聚合物是高分子化学领域的一个重要研究方向. 在基 于三键的聚合反应开发方面, 目前已成功通过单炔烃 $\left(\mathrm{RC} \equiv \mathrm{CR}^{\prime}\right)$ 的易位聚合方法制备出了一系列线型多烯. 这些多烯表现出了诸多独特的性质, 比如液晶性、光电
导性、电致发光、螺旋手性、超分子自组装以及生物活 性等 ${ }^{[1]}$. 通过不同双炔烃的环三聚可以得到多种超支化 的聚芳烃. 这些聚芳烃具有可加工性、高折射率、高热 稳定性、在激光脉冲的激发下可表现出非线性光学活性 等许多优良性质 ${ }^{[2]}$.

然而，目前所开发出的大多数聚合物虽然在其稀溶

$\dagger$ Han, Ting and Cathy K. W. Jim contributed equally to this work. 韩婷和詹嘉慧对本文工作贡献相同.

*E-mail: chjacky@ust.hk, tangbenz@ust.hk

Received July 16, 2016; published August 12, 2016.

Supporting information for this article is available free of charge via the Internet at http://sioc-journal.cn.

Project supported by the National Basic Research Program of China (973 Program; 2013CB834701, 2013CB834702), the University Grants Committee of Hong Kong (AoE/P-03/08), the National Natural Science Foundation of China (21490570, 21490574), the Research Grants Council of Hong Kong (604913, 16305014 and 16303815), and the Innovation and Technology Commission (ITC-CNERC14S01). B.Z.T. thanks the support of the Guangdong Innovative Research Team Program (201101C0105067115).

项目受国家基础研究发展规划项目(973 项目; 2013CB834701, 2013CB834702), 香港大学教育资助委员会(AoE/P-03/08), 国家自然科学基金(21490570 和 21490574), 香港研究资助局 $(604913,16305014,16303815)$ 及创新科技署(ITC-CNERC14S01)资助. 同时感谢广东省创新科研团队项目 (201101C0105067115)资助. 
液中发光良好, 但在固态下却发光很弱甚至不发光, 这 种在凝聚相中由于聚集体的生成而猝灭发光的现象叫 做“聚集猝灭荧光(Aggregation-caused quenching, ACQ)” 现象 ${ }^{[3]}$. 该效应在一定程度上阻碍了聚合物在光电领域 的实际应用. 2001 年, 唐本忠课题组提出了一种与传统 苂光分子的 ACQ 性质截然相反的概念: “聚集诱导发光 (Aggregation-induced emission, AIE)” 效应 ${ }^{[4]}$. 一些在溶 液态下不发光的分子，比如四苯基乙烯 (Tetraphenylethylene, TPE)、硅杂环戊二烯、亚甲基环戊 二烯类化合物, 可通过形成聚集体诱导其高效发光. AIE 效应的发现大大拓展了苂光分子在实际生活中的应 用范围, 比如用作含水介质中的化学传感器、生物探针、 刺激响应材料及有机发光二极管器件中的发光层等 ${ }^{[5]}$. 在已开发的 AIE 发光分子当中, TPE 因其合成简单, 易 功能化及高苂光量子产率 $\left(\Phi_{\mathrm{F}}\right)$ 等优点得到了广泛的研 究关注.

在实际应用中，低分子量的发光体必须要经过真空 气相沉积过程等昂贵的工艺被制备成固态薄膜形式来 使用, 这并不适合大面积平板设备的生产制造. 解决这 一问题的途径之一是合成高分子量的聚合物, 仅需通过 旋转涂膜和静态浇注等简单的加工技术即可将其制备 成大面积薄膜. 因此, 开发具有 AIE 特性的聚合物十分 必要 ${ }^{[6]}$. 在前期工作中, 我们已经通过叠氮和炔的“点击 聚合” 反应成功制备出了含 TPE 基元的具有 AIE 活性 的聚三唑 ${ }^{[7]}$. 然而, 为了得到高分子量的聚合物, 此双 组分聚合反应必须满足两单体的等当量条件. 相比之
下，通过 $\mathrm{A}_{2}$-类型的双炔烃单体的均聚反应来获得高分 子量的 AIE 聚合物则可避免这一问题，同时简化实验操 作.

本文设计合成了几种含 TPE 基元的双炔烃单体，通 过其在 Glaser-Hay 反应条件下 ${ }^{[8]}$ 的偶联聚合反应成功制 备出了具有 AIE 活性的新型聚合物, 对单体和聚合物的 结构进行了充分表征, 并对其光学性质进行了研究.

\section{2 结果与讨论}

\section{1 单体制备}

为丰富 $\mathrm{AIE}$ 聚合物种类，含有 TPE 基元的双炔烃分 子 $1(m)$ 和 2 通过图式 1 所示的合成路线得到. 起始化合 物 $\mathbf{3}(m)$ 和 6 按照已发表的步骤合成 ${ }^{[7 b, 8 c]}$. 从原料 $\mathbf{3}(m)$ 和 4-碘苯酚出发, 经醚化反应生成中间化合物 $4(m)$, 之后 $\mathbf{4}(m)$ 和三甲基硅炔在 $\mathrm{PdCl}_{2}\left(\mathrm{PPh}_{3}\right)_{2}$ 和 $\mathrm{CuI}$ 的催化下发生 偶联反应得到 $\mathbf{5}(m)$. 在 $\mathrm{KOH}$ 的四氢呋喃 $(\mathrm{THF})$ 甲醇混 合溶液中, $\mathbf{5}(m)$ 发生脱硅反应，高产率地产生 $\mathbf{1}(m)$ 单体. 双炔烃 2 通过原料 6 和炔丙基溴的亲核取代反应制得. 所有的中间产物和单体都使用标准的光谱技术进行了 表征分析, 所得结果均与预期分子结构相符合(具体实 验步骤和结构表征数据见实验部分).

\section{2 聚合物合成}

图式 2 为聚合物 $\mathrm{P} 1(m)$ 和 $\mathrm{P} 2$ 的合成路线, 研究发现 单体 $\mathbf{1}(\mathrm{m})$ 或 2 在 $\mathrm{CuCl}$ 和 $N, N, N^{\prime}, N^{\prime}$-四甲基乙二胺 (TMEDA)的存在下可以高效地发生偶联聚合反应，生
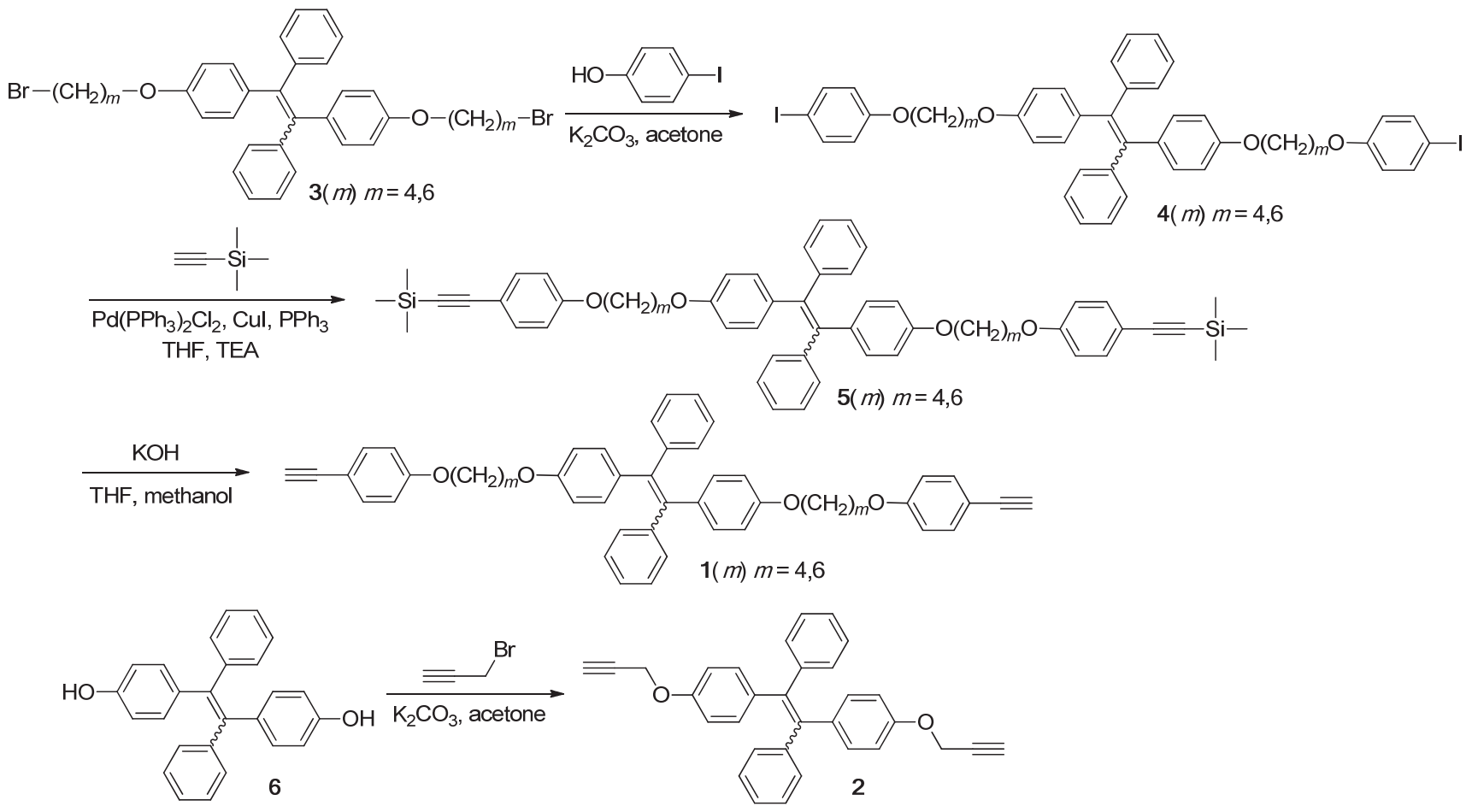

图式 1 单体 $\mathbf{1}(\mathrm{m})$ 和 $\mathbf{2}$ 的合成路线

Scheme 1 Synthetic route to monomers $\mathbf{1}(m)$ and $\mathbf{2}$ 


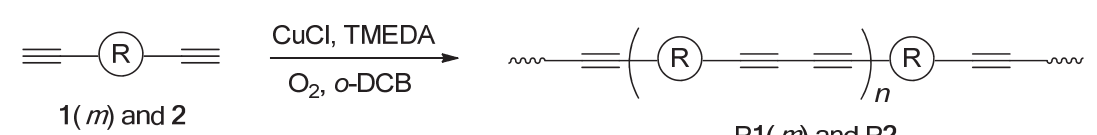

$\mathrm{P} 1(m)$ and $\mathrm{P} 2$

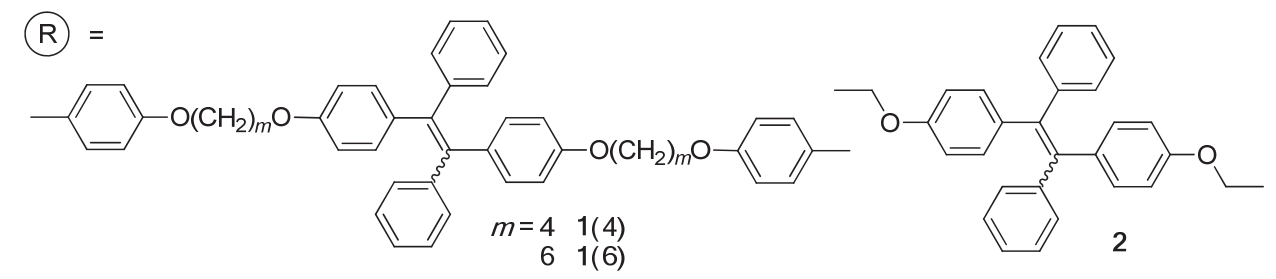

图式 2 聚合物 P1 $(m)$ 和 P2 的合成路线

Scheme 2 Synthetic route to polymers $\mathrm{P} 1(m)$ and P2

成高分子量的线型聚炔.

如表 1 所示, 我们首先尝试了单体 1(4)的聚合反应, 结果表明: 1(4)在 $\mathrm{CuCl}$ 和 TMEDA 的存在下以邻二氯苯 $(o-\mathrm{DCB})$ 为溶剂在 $60{ }^{\circ} \mathrm{C}$ 下反应 $3 \mathrm{~h}$ 可高产率地得到聚合 物 P1(4), $M_{\mathrm{w}}$ 为 18300 (表 1, Run 1). 在与 1(4)相同的反应 条件下, 单体 1(6)和 2 也可以较高的产率得到相近分子 量的聚合物, P1(6)和 P2 的分子量分别为 14800 和 17600 .

表 $\mathbf{1}$ 二炔烃 $\mathbf{1}(m)$ 和 $\mathbf{2}$ 的聚合反应 ${ }^{a}$

Table 1 Polymerizations of diynes $\mathbf{1}(\mathrm{m})$ and $\mathbf{2}^{a}$

\begin{tabular}{ccccc}
\hline Run & Monomer & Yield $/ \%$ & $M_{\mathrm{w}}{ }^{b}$ & $M_{\mathrm{w}} / M_{\mathrm{n}}{ }^{b}$ \\
\hline 1 & $\mathbf{1}(4)$ & 92.9 & 18300 & 1.9 \\
2 & $\mathbf{1}(6)$ & 86.7 & 14800 & 1.7 \\
3 & $\mathbf{2}$ & 71.9 & 17600 & 2.7 \\
\hline
\end{tabular}

${ }^{a}$ Carried out at $60{ }^{\circ} \mathrm{C}$ in $o$-dichlorobenzene for $3 \mathrm{~h}$ with an air stream bubbling through the reaction mixtures; $[\mathrm{M}]_{\mathrm{o}}=50 \mathrm{mmol} / \mathrm{L} ;[\mathrm{CuCl}]=2.5 \mathrm{mmol} / \mathrm{L}$, $[$ TMEDA $]=8.6 \mathrm{mmol} / \mathrm{L} .{ }^{b}$ Determined by GPC in THF on the basis of a linear polystyrene calibration.

\section{3 结构表征}

单体和聚合产物的化学结构使用红外和核磁共振 光谱技术进行了充分的表征和比对, 分析表明产物结构 与预期结构完全相符. 以聚合物 P1(6)及其单体 1(6)为 例, 其红外光谱对比如图 1 所示. 可以看到, 在反应后, 单体 1(6)的红外谱图中 $3286 \mathrm{~cm}^{-1}$ 处的 $\equiv \mathrm{C}-\mathrm{H}$ 伸缩振动 和 $2106 \mathrm{~cm}^{-1}$ 处的 $\mathrm{C} \equiv \mathrm{C}$ 伸缩振动吸收峰在聚合物 $\mathrm{P} 1(6)$ 的谱图中均完全消失, 取而代之的是在 2212 和 2139 $\mathrm{cm}^{-1}$ 处出现了 $\mathrm{C} \equiv \mathrm{C}-\mathrm{C} \equiv \mathrm{C}$ 的振动吸收峰, 证实了此偶 联聚合反应的发生.

图 2 给出了单体 1(6)及其聚合物 P1(6)在氞代氯仿 中的核磁共振氢谱对比谱图. 图 2A 中化学位移 $\delta 2.99$ 处为 1(6)结构中炔氢的特征共振峰, 在反应后该峰完全 消失. 通过和单体 1(6)的图谱对比可以很容易地得到 P1(6)图谱中各共振峰的归属. 没有其他非预期结构的 信号被检测到, 表明所得聚合产物 P1(6)的确为图 2B 所 示的分子结构.

为进一步表征聚合物结构, 又进行了 ${ }^{13} \mathrm{C} \mathrm{NMR}$ 谱 图分析(图 3). 在 P1(6)的核磁共振碳谱中观察不到单体 1(6)中端炔碳在化学位移 $\delta 83.7$ 和 75.7 处的特征共振峰,

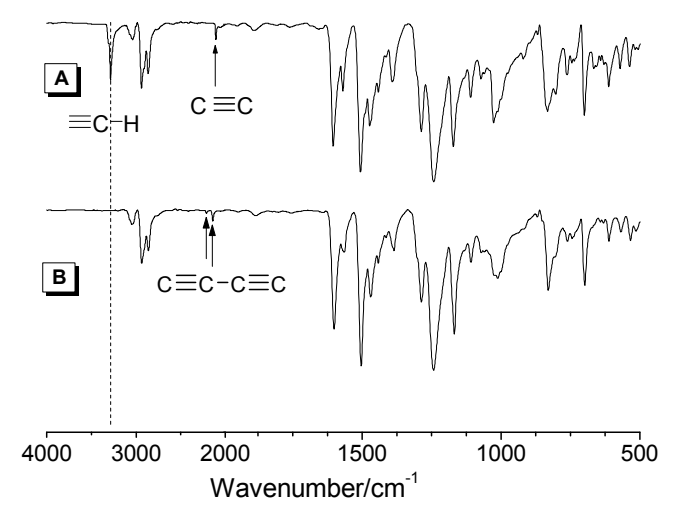

图 1 (A)单体 1(6)和(B)聚合物 P1(6)的红外图谱

Figure 1 IR spectra of (A) monomer 1(6) and (B) its polymer P1(6)
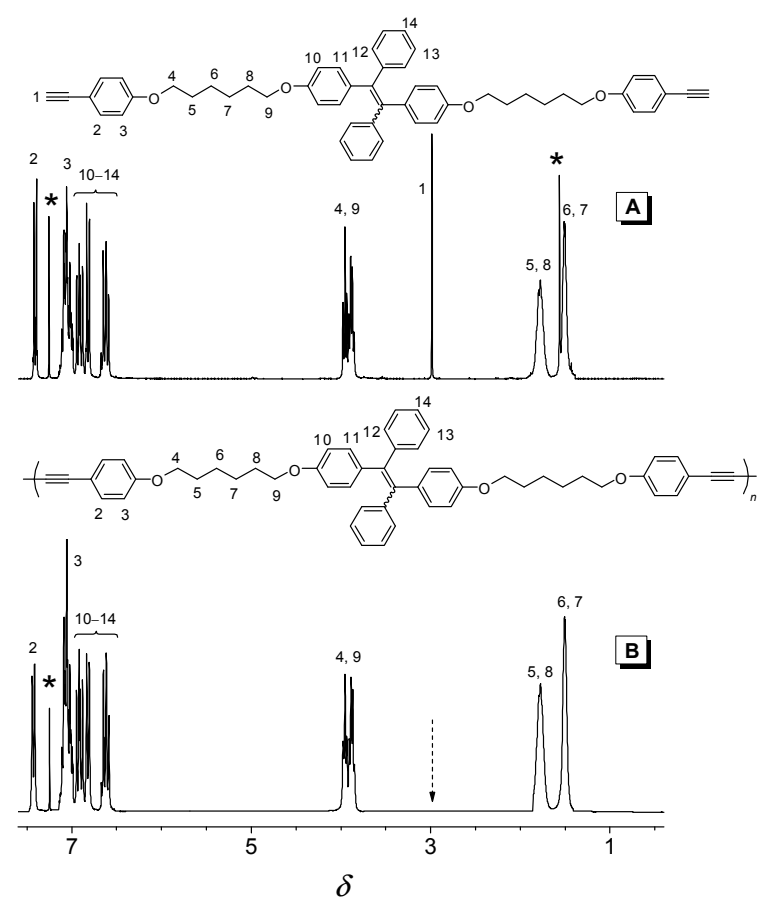

图 2 (A)单体 $1(6)$ 和(B)聚合物 P1(6)溶解在氛代氯仿中的核磁共振氢 谱; 溶剂峰和水峰以星号进行标记

Figure $2{ }^{1} \mathrm{H}$ NMR spectra of (A) monomer 1(6) and (B) polymer P1(6) in $\mathrm{CDCl}_{3}$. The solvent and water peaks are marked with asterisks 
而在 $\delta 81.3$ 和 72.9 处观察到了新出现的联炔烃碳的共振 峰, 说明单体 1(6)中的端炔三键已通过偶联聚合反应被 转化为 $\mathrm{P1}(6)$ 的内炔键.

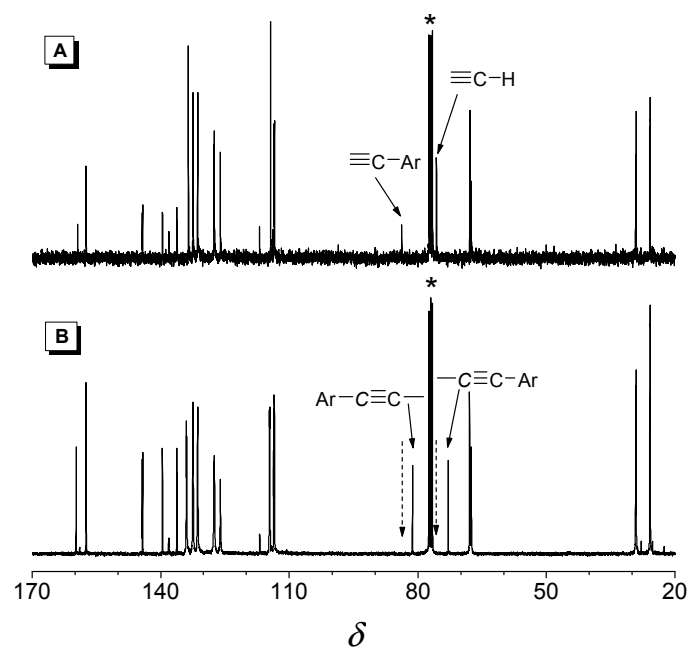

图 3 (A)单体 1(6)和(B)聚合物 P1(6)溶解在氞代氯仿中的核磁共振碳 谱; 溶剂峰以星号进行标记

Figure $3{ }^{13} \mathrm{C}$ NMR of (A) monomer 1(6) and (B) its polymer P1(6) in $\mathrm{CDCl}_{3}$. The solvent peaks are marked with asterisks

\subsection{UV-vis 吸收光谱}

图 4 为单体 $\mathbf{1}(6) 、 \mathbf{2}$ 及聚合物 $\mathrm{P} 1(m)$ 和 $\mathrm{P} 2$ 在 $\mathrm{THF}$ 溶液中的 UV-vis 吸收光谱. 两单体的吸收峰中心均出 现在约 $320 \mathrm{~nm}$ 处, 该吸收峰与 TPE 基元的 $\pi-\pi$ *跃迁有 关. P2 表现出了与之类似的光谱峰形, 而 P1 $(m)$ 则在 340 $\mathrm{nm}$ 处出现了新的吸收峰, 可能是由于聚合物中新形成 的联炔烃基团将苯环连接起来造成的. 值得注意的是所 有聚合物均具有高度透光性, 可接近 $100 \%$ 地透过整个 可见光区的光，有希望应用于先进光学材料当中.

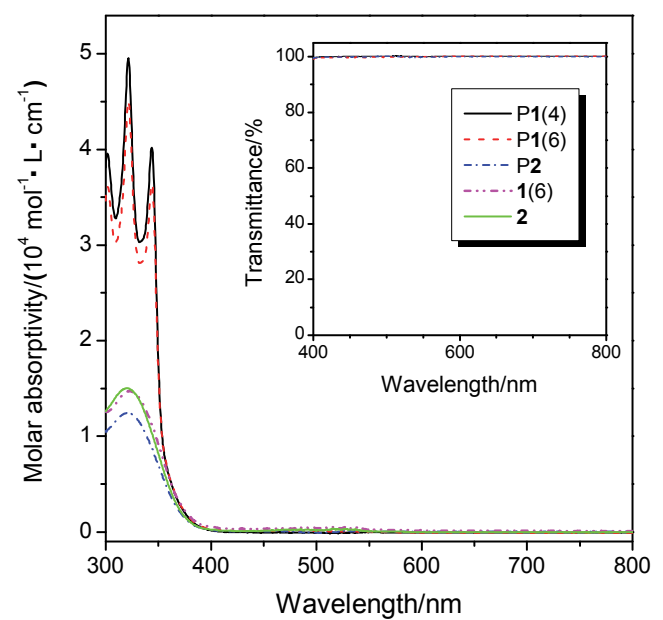

图 4 1 $(6) 、 2$ 及其聚合物 $\mathrm{P} 1(m) 、 \mathrm{P} 2$ 在 $\mathrm{THF}$ 溶液 $(10 \mu \mathrm{mol} / \mathrm{L})$ 中的吸 收光谱. 插图: 聚合物在 THF 溶液中的透射光谱

Figure 4 Absorption spectra of THF solutions $(10 \mu \mathrm{mol} / \mathrm{L})$ of $\mathbf{1}(6)$ and $\mathbf{2}$ and their polymers $\mathbf{P} \mathbf{1}(\mathrm{m})$ and $\mathbf{P 2}$. Inset: Transmittance spectra of the polymers in THF solutions

\section{5 聚集诱导发光特性}

TPE 具有典型的 AIE 特性: 当其以分子形式分散在 良溶剂中时几乎不发光，但是当在不良溶剂中发生聚集 或者被制备成固态薄膜形式时则会变得强烈发光. 为了 探究含有 TPE 基元的聚炔是否具有类似的性质, 我们研 究了所得聚炔在溶液态和聚集态下的发光行为. 聚集体 是通过在剧烈摚拌下向聚合物的 THF 溶液中加水制得. 得到的混合体系表观看起来均一透明，说明聚合物聚集 体处于纳米尺寸. 当用波长为 $345 \mathrm{~nm}$ 的光激发时, P1(6) 的 $\mathrm{THF}$ 稀溶液的发光光谱几乎是一条平行于横坐标的 直线, 证明 P1(6)在溶液态下发光很弱(图 5A). 然而, 在 相同测试条件下，当向其溶液中加入大量水时，可以明 显观察到其发光光谱在约 $480 \mathrm{~nm}$ 处出现强荧光发射峰. 由于水是 P1(6)的非溶剂, 因此在高含水量的 $\mathrm{THF} /$ 水混
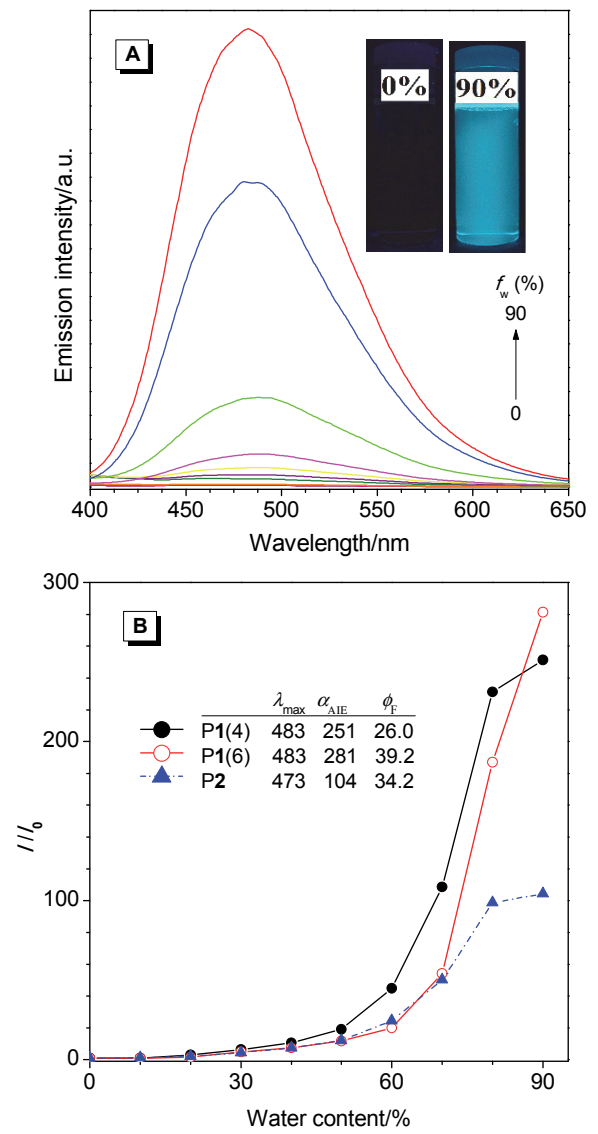

图 5 (A) P1(6)在 $\mathrm{THF} /$ 水混合溶剂中的发射光谱. 浓度: $10 \mu \mathrm{mol} / \mathrm{L}$; 激发波长: $345 \mathrm{~nm}$. (B) P1 $(m)$ 和 $\mathrm{P} 2$ 在最大发射波长 $\left(\lambda_{\max }\right)$ 处的 $I / I_{0}$ 值随 含水量组成的变化曲线. AIE 系数 $\left(\alpha_{\mathrm{AIE}}\right)$ 定义为 $I_{90} / I_{0}$, 其中 $I_{90}$ 和 $I_{0}$ 分别 指聚合物在含水量为 $90 \%$ 和 $0 \%$ 的 $\mathrm{THF} /$ 水混合溶剂中的在 $\lambda_{\max }$ 处的发 光强度. $\Phi_{\mathrm{F}}$ 为积分球测得的聚合物薄膜的荧光量子产率

Figure 5 (A) Emission spectra of P1(6) in THF/water mixtures. Concentration: $10 \mu \mathrm{mol} / \mathrm{L}$; excitation $345 \mathrm{~nm}$. (B) Plot of $I / I_{0}$ values of P1 $(m)$ and $\mathrm{P} 2$ at the maximum emission wavelength $\left(\lambda_{\max }\right)$ versus the compositions of the aqueous mixtures. AIE factor $\left(\alpha_{\mathrm{AIE}}\right)$ defined as $I_{90} / I_{0}$, where $I_{90}$ and $I_{0}$ are the emission intensities at the $\lambda_{\max }$ in the THF/water mixtures with $90 \%$ and $0 \%$ water contents, respectively. $\Phi_{\mathrm{F}}=$ fluorescent quantum yield of the polymer thin film determined by an integrating sphere 
合溶液中聚合物链必然会发生聚集, 进而诱导 P1(6)开 始发光, 表现出 AIE 活性. 聚合物 P1(4)和 P2 也表现出 了类似的发光行为. 这些聚合物的 AIE 行为可以用 TPE 结构的 “分子内旋转受限” 机理来解释. 即使 TPE 被引 入到聚炔结构中, 其苯环在 THF 溶液中仍可自由旋转, 因此聚合物在稀溶液态下几乎不发光. 而在聚集状态 下, 分子内苯环的自由旋转受到了限制, 抑制了激发态 的非辐射失活途径, 从而促使聚合物开始发光.

通过比较聚合物溶液及其聚集体在最大发射波长 处的发光强度可以得到聚炔的 AIE 系数 $\left(\alpha_{\mathrm{AIE}}=I_{90} / I_{0}\right)$ (图 5B). P1(6)表现出了最大的 $\alpha_{\mathrm{AIE}}$ 值(约 280), 其他聚合物 的苂光增强倍数也都大于 100 倍. 聚合物粉末在紫外光 照射下均可强烈发光(图 S1). 聚合物 P1(4)、P1(6)和 P2 薄膜的量子产率通过积分球测得，分别为 $26.0 \% 、 39.2 \%$ 和 $34.2 \%$.

\section{6 溶解性和热稳定性}

所有的聚炔产物均可完全溶解于常见的有机溶剂 当中, 比如甲苯、THF、氯仿和二氧六环, 因此很容易 通过旋涂和静态浇注等过程被制备成坚硬的固态薄膜. 同时这类聚合物还具有很高的热稳定性. 如图 6 所示, 在氮气保护下，聚合物失重 $5 \%$ 时的热分解温度 $\left(T_{\mathrm{d}}\right)$ 均高 于 $370{ }^{\circ} \mathrm{C}$, 说明其具有强耐热性. 在所有的聚合物当 中, P2 因其较高的芳烃含量, 在 $800{ }^{\circ} \mathrm{C}$ 高温下仍可保留 超过 $50 \%$ 的残炭量. 图 S2 所示的差示扫描量热 (Differential scanning calorimetry, DSC)测试结果表明: 聚合物 P1(4), P1(6)和 P2 的玻璃化转变温度 $\left(T_{\mathrm{g}}\right)$ 值分别 为 120,121 和 $119{ }^{\circ} \mathrm{C}$.

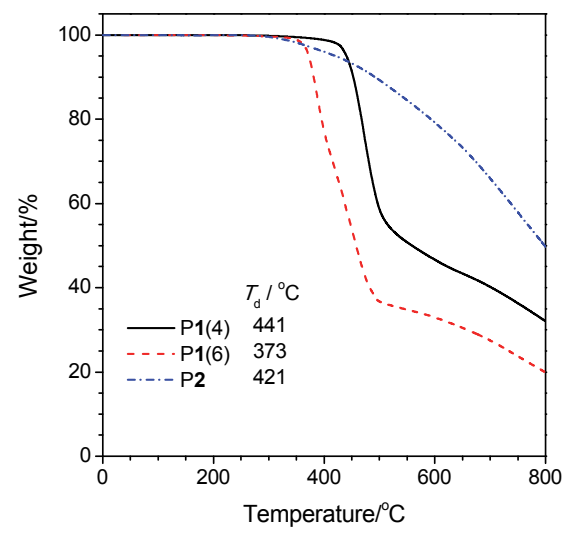

图 $6 \mathrm{P} 1(m)$ 和 $\mathrm{P} 2$ 在氮气条件下的 TGA 曲线, 加热速率 $10{ }^{\circ} \mathrm{C} / \mathrm{min} . T_{\mathrm{d}}$ 指失重 $5 \%$ 时的温度

Figure 6 TGA thermograms of $\mathrm{P} 1(m)$ and $\mathrm{P} 2$ recorded under nitrogen at a heating rate of $10{ }^{\circ} \mathrm{C} / \mathrm{min} . T_{\mathrm{d}}=$ temperature for $5 \%$ weight loss

\section{7 光折射}

高折射率聚合物材料因其在抗反射涂层、光波导、 记忆以及全息图像记录系统等领域的潜在实际应用价 值而受到广泛关注. 与其他具有高折射率的材料相比, 高折射率聚合物材料具有质量轻、加工性能好和抗冲击 性等优势 ${ }^{[9]}$. 已有研究报道超支化聚炔由于大量可极化
芳香环和联炔烃基团的存在而表现出了较高的折射率 $(n)^{[10]}$. 本文合成出的线型聚炔同样具有此性质. 如图 7 所示, P1(6)在宽光谱区域(400 1700 nm)处表现出高折 射率 $(n=1.7787 \sim 1.6543)$, 此折光指数值要远高于已经 商业化的重要光学塑料, 例如聚甲基丙烯酸甲酯 $(n=$ $1.49)$, 聚对苯二甲酸乙二酯 $(n=1.59)$, 聚碳酸酯 $(n=$ $1.59)$ 等. 在 P1(6)的固态薄膜中几乎未观察到任何双折 射现象, 同时在图 S3 所示的 P1(6)的固态薄膜的 X 射线 衍射(X-ray diffractometry, XRD)结果中没有检测到衍射 峰, 表明其无定型性质 ${ }^{[11]}$. 这类聚合物白色的外观结合 其高折光指数使其在需要高折射率透明材料的光学领 域中有着潜在的应用前景 ${ }^{[12]}$.

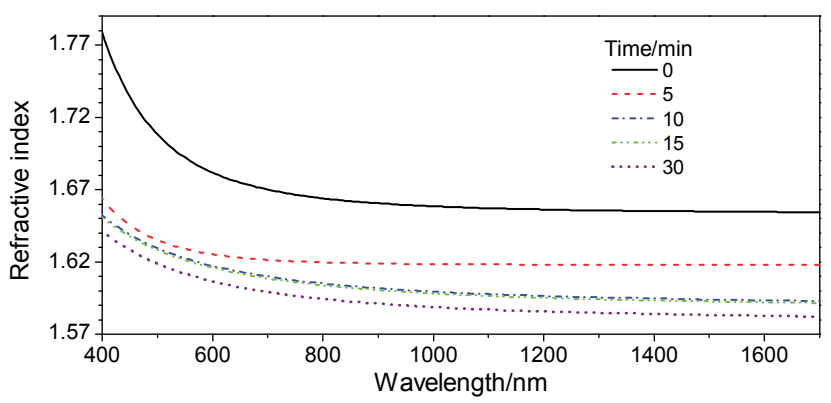

图 7 P1(6)薄膜经不同 UV 照射时间后的折射率变化

Figure 7 Change in the refractive index of thin film of P1(6) by UV irradiation at different exposure times

进一步的研究发现聚合物薄膜的折射率可通过 UV 照射方式来进行调控. 从图 7 可看出: 一经曝露在 UV 光下, P1(6)在整个光谱区域(400 $1700 \mathrm{~nm})$ 范围内的 $n$ 值均发生下降. 聚合物薄膜在电磁通讯重要波段 1550 $\mathrm{nm}$ 处的折光指数随 UV 光照时间的变化呈现出一阶指 数衰减(图 8). P1(6)未经处理的样品和 UV 光照了 $30 \mathrm{~min}$ 的样品之间的折射率相差 $(\Delta n)$ 可达 0.072 , 说明这种简 单的 UV 光照技术可以有效地调节此类聚合物的折射率 大小，使之发生大幅度改变.

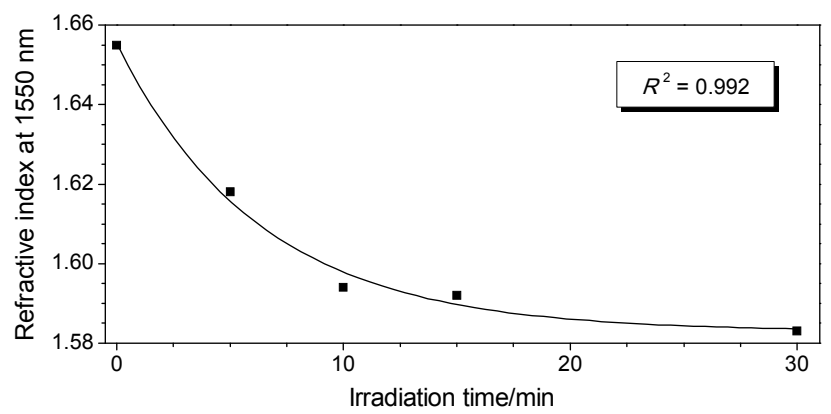

图 $8 \mathrm{P} 1(6)$ 在 $1550 \mathrm{~nm}$ 处的折射率随 UV 光照时间的变化曲线 Figure 8 Plot of refractive index of P1(6) at $1550 \mathrm{~nm}$ versus the UV exposure time

\section{8 色散}

对于光学材料来说，低色差是其在实际应用中的另 一个重要要求. 阿贝数可用来衡量材料的折射率随波长 
的变化(色散)情况，其值定义为:

$$
v_{\mathrm{D}}=\frac{n_{\mathrm{D}}-1}{n_{\mathrm{F}}-n_{\mathrm{C}}}
$$

其中 $n_{\mathrm{D}}, n_{\mathrm{F}}$ 和 $n_{\mathrm{C}}$ 分别是在 Fraunhofer $\mathrm{D}, \mathrm{F}$ 和 $\mathrm{C}$ 光谱线处, 即在 $589.2,486.1$ 和 $656.3 \mathrm{~nm}$ 处的折光率值. 利用在无 吸收波长范围内的 1064,1319 和 $1550 \mathrm{~nm}$ 处的 $n$ 值我们 计算了修正的阿贝数 ( $\left.v_{\mathrm{D}}^{\prime}{ }^{\prime}\right)$ 来评估其作为光学材料的实 际应用潜力 ${ }^{[13]}$. 前两个波长是基于商业激光波段 $(\mathrm{Nd}: \mathrm{YAG})$ 的实际应用意义而选择的, 而最后一个波长 对应的是电磁通讯波段. 修正的阿贝数被定义为:

$$
v_{\mathrm{D}}^{\prime}=\frac{n_{1319}-1}{n_{1064}-n_{1550}}
$$

其中 $n_{1319}, n_{1064}$ 和 $n_{1550}$ 分别为在 1319,1064 和 $1550 \mathrm{~nm}$ 处的 $n$ 值. 色散 $\left(D^{()}\right)$为阿贝数值的倒数 $\left(v_{\mathrm{D}}{ }^{\left.()^{\prime}\right)}\right.$ :

$$
D^{()}=\frac{1}{v_{D}^{()}}
$$

表 2 列出了 P1(6)薄膜经过不同 UV 光照时间后的 折光指数和光学色散数值. 其在 UV 光照 $5 \mathrm{~min}$ 后的 $v_{\mathrm{D}}$ 和 $v_{D}^{\prime}$ 值分别为 42 和 3090 , 对应的 $D$ 和 $D^{\prime}$ 值低至 0.024 和 0.0003 . 优异的色散性能使得这类聚合物成为制备先 进光学器件的潜在材料 ${ }^{[14]}$.

表 2 P1(6)的折光指数和光学色散 ${ }^{a}$

Table 2 Refractive indices and chromatic dispersions of P1 $(6)^{a}$

\begin{tabular}{cccccc}
\hline$t^{b} / \min$ & $n_{1550}$ & $\nu_{\mathrm{D}}$ & $V_{\mathrm{D}}{ }^{\prime}$ & $D$ & $D^{\prime}$ \\
\hline 0 & 1.655 & 16.9 & 226 & 0.059 & 0.0044 \\
5 & 1.618 & 42.0 & 3090 & 0.024 & 0.0003 \\
10 & 1.594 & 32.2 & 126 & 0.031 & 0.0079 \\
15 & 1.592 & 32.1 & 126 & 0.031 & 0.0079 \\
30 & 1.583 & 31.6 & 124 & 0.032 & 0.0080 \\
\hline
\end{tabular}

${ }^{a}$ All data taken from Figure 8. Abbreviations: $n=$ refractive index, $v_{\mathrm{D}}=$ Abbé number, $v_{\mathrm{D}}{ }^{\prime}=$ modified Abbé number, $D=$ chromatic dispersion in the visible region, and $D^{\prime}=$ chromatic dispersion in the IR region. ${ }^{b} \mathrm{UV}$ exposure time.

\section{9 光刻蚀图案化}

在光刻蚀等高科技工艺中, 光敏感的聚合物被作为 光刻胶广泛使用. 对于制造光、电激发的器件和生物传 感、探测系统来说, 产生苂光成像是很关键的部分 ${ }^{[15]}$. 本文所合成的线型聚炔结构中含有大量光敏感的联炔 烃基团，使其在 UV 照射下很容易发生光交联反应，从 而可作为光刻胶材料用来生成光刻蚀图案. 再结合这类 聚炔的固态发光性质和良好的可加工性能, 使其成为一 种可通过光刻过程来产生苂光图案的优异材料. 如图 9 所示, P1(6)的固态薄膜在空气中用 UV 光经铜制光掩膜 照射 $5 \mathrm{~min}$, 曝露在 UV 光下的部分(网格线)发光被猝灭, 而未曝露的方形区域则仍保持发光, 从而生成一个高分 辨率的正向二维苂光图案. 进一步将该图案在 1,2-二氯 乙烷中浸泡后, 之前未曝露于 UV 光下的部分将被溶解 洗去, 留下的为发生交联的不能被溶解掉的部分, 从而 得到一个具有清晰边缘的反向三维光刻图案.
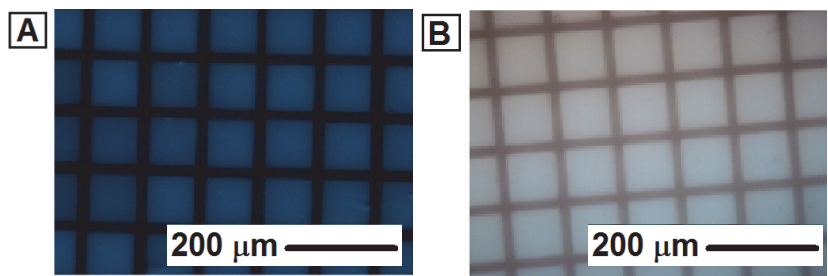

图 $9 \mathrm{P} 1(6)$ 经紫外光照射 $5 \mathrm{~min}$ 后生成的(A)二维荧光图案和(B)反向 三维荧光图案. 照片在(A)紫外光照射和(B)一般室内光线下拍摄

Figure 9 (A) Two-dimensional fluorescent image and (B) three-dimensional negative photopattern generated by UV irradiation of P1(6) for 5 minutes. The photographs were taken under (A) UV illumination and (B) normal room light

\section{3 结论}

在本文工作中，合成了几种含 TPE 基元的双炔烃单 体，其偶联聚合反应在以邻二氯苯作溶剂、 $\mathrm{CuCl}$ 和 TMEDA 存在的条件下可以顺利进行，可高产率地生成 线型聚炔. 所得聚合物均具有良好的溶解性、成膜性和 热稳定性. 同时还具有高度透光性, 其薄膜表现出高折 射率和低色差, 并且其折射率数值可通过简单的调节 UV 照射时间来进行控制. 此外, 这类聚炔还具有光响 应性，其薄膜经 UV 照射很容易发生光交联化，生成高 分辨率的二维和三维荧光图案. 这些独特的性质使得此 类聚合物在许多高科技领域当中有着潜在的应用前景.

\section{4 实验部分}

\section{1 单体的合成}

4.1.1 中间体 1,2-双[4-(4-碘代苯基烷氧基)苯基]-1,2-二 苯基乙烯 $[4(m)]$ 的合成

如图式 2 所示, 以 1,2-双[4-(4-碘代苯基丁氧基)苯 基]-1,2-二苯基乙烯[4(4)]的合成步骤为例, 在 $500 \mathrm{~mL}$ 圆 底烧瓶中加入 $3.17 \mathrm{~g}$ (5 mmol) 1,2-双[4-(4-溴代丁氧基) 苯基]-1,2-二苯基乙烯[3(4)], $3.3 \mathrm{~g}$ (15 mmol) 4-碘苯酚, $3.5 \mathrm{~g}(25 \mathrm{mmol})$ 碳酸钾和 $200 \mathrm{~mL}$ 丙酮, 加热至回流, 过 夜反应. 反应停止后过滤出固体, 滤液旋蒸浓缩. 粗产 物通过硅胶色谱柱分离提纯 [淋洗液: $V$ (氯仿) $: V($ 正己 烷 $)=1: 2]$, 得到浅黄色油状产物, 产率 $85.9 \% .{ }^{1} \mathrm{H}$ NMR $\left(300 \mathrm{MHz}, \mathrm{CDCl}_{3}\right) \delta: 7.53(\mathrm{~d}, 4 \mathrm{H}), 7.06(\mathrm{~m}, 10 \mathrm{H})$, $6.88(\mathrm{~m}, 4 \mathrm{H}), 6.65(\mathrm{~m}, 8 \mathrm{H}), 3.97(\mathrm{~m}, 8 \mathrm{H}), 1.92(\mathrm{~m}, 8 \mathrm{H})$. ${ }^{13} \mathrm{C}$ NMR $\left(75 \mathrm{MHz}, \mathrm{CDCl}_{3}\right) \delta: 158.8,157.3,144.3,139.6$, $138.2,136.4,136.3,132.5,131.4,127.6,127.5,126.1$, $116.9,113.6,113.5,82.6,67.6,67.1,25.9$. 中间体 1,2-双 [4-(4-碘代苯基已氧基)苯基]-1,2-二苯基乙烯 [4(6)]以 $3.45 \mathrm{~g}$ (5 mmol) 1,2-双[4-(4-溴代已氧基)苯基]-1,2-二苯 基乙烯 $[3(6)]$ 采用上述相同步骤得到，为浅黄色液体， 产率 88.2\%. ${ }^{1} \mathrm{H}$ NMR $\left(300 \mathrm{MHz}, \mathrm{CDCl}_{3}\right) \delta: 7.54(\mathrm{~d}, 4 \mathrm{H})$, $7.06(\mathrm{~m}, 10 \mathrm{H}), 6.92(\mathrm{~m}, 4 \mathrm{H}), 6.64(\mathrm{~m}, 8 \mathrm{H}), 3.88(\mathrm{~m}, 8 \mathrm{H})$, $1.50(\mathrm{~m}, 8 \mathrm{H}), 1.33(\mathrm{~m}, 8 \mathrm{H}) .{ }^{13} \mathrm{C} \mathrm{NMR}\left(75 \mathrm{MHz}, \mathrm{CDCl}_{3}\right)$ 
$\delta: 158.9,157.4,144.3,144.2,139.6,138.1,136.3,136.2$, $132.5,131.4,127.6,127.5,126.1,116.9,113.6,113.5$, $82.4,67.9,67.5,29.2,29.1$.

4.1.2 中间体 1,2-双[4-(4-三甲基硅乙炔基苯基烷氧基) 苯基]-1,2-二苯基乙烯 $[\mathbf{5}(\mathrm{m})]$ 的合成

以 1,2-双 [4-(4-三甲基硅乙炔基苯基丁氧基)苯 基]-1,2-二苯基乙烯 [5(4)]的合成步骤为例, 在配有搅拌 子和橡胶塞的 $500 \mathrm{~mL}$ 圆底烧瓶中加入 $4.56 \mathrm{~g} \mathrm{(5} \mathrm{mmol)}$ 4(4), $71 \mathrm{mg}$ (0.1 mmol) $\mathrm{Pd}\left(\mathrm{PPh}_{3}\right)_{2} \mathrm{Cl}_{2}, 19 \mathrm{mg}(0.1 \mathrm{mmol})$ $\mathrm{CuI}$, 和 $26 \mathrm{mg}$ (0.1 mmol) $\mathrm{PPh}_{3}$, 之后用注射器加入干燥 的 $\mathrm{Et}_{3} \mathrm{~N}(100 \mathrm{~mL})$ 和 THF $(100 \mathrm{~mL})$. 边搅拌边加入 2.1 $\mathrm{mL}$ (15 mmol, $1.48 \mathrm{~g})$ 三甲基硅乙炔, 混合物在室温下搅 拌 12 h. 抽滤收集沉淀物, 用乙醚洗涤. 收集滤液, 减 压除去其中的溶剂, 粗产物过硅胶柱 [淋洗液: $V($ 氯 仿 $): V$ (正己烷 $)=1: 2]$ 纯化后得到浅黄色油状产物, 产 率为 79.2\%. ${ }^{1} \mathrm{H} \mathrm{NMR}\left(300 \mathrm{MHz}, \mathrm{CDCl}_{3}\right) \delta: 7.40,(\mathrm{~d}, 4 \mathrm{H})$, $7.07(\mathrm{~m}, 10 \mathrm{H}), 7.02(\mathrm{t}, 4 \mathrm{H}), 6.80(\mathrm{~d}, 4 \mathrm{H}), 6.62(\mathrm{t}, 4 \mathrm{H}), 3.97$ $(\mathrm{m}, 8 \mathrm{H}), 1.93(\mathrm{~m}, 8 \mathrm{H}), 0.23(\mathrm{~s}, 18 \mathrm{H}) .{ }^{13} \mathrm{C} \mathrm{NMR}(75 \mathrm{MHz}$, $\left.\mathrm{CDCl}_{3}\right) \delta: 159.8,158.0,145.0,144.9,140.3,137.1,137.0$, $134.1,133.2,132.1,128.3,128.2,126.8,115.8,115.0$, 114.3，114.2，105.9，93.1，68.2，67.8，26.6，0.8. 中间体 1,2-双[4-(4-三甲基硅乙炔基苯基己氧基)苯基]-1,2-二苯

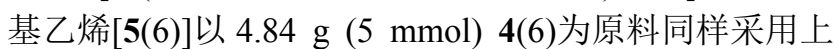
述步骤得到, 为浅黄色液体, 产率 75.3\%. ${ }^{1} \mathrm{H}$ NMR (300 $\left.\mathrm{MHz}, \mathrm{CDCl}_{3}\right) \delta: 7.40(\mathrm{~d}, 4 \mathrm{H}), 7.07(\mathrm{~m}, 10 \mathrm{H}), 6.92(\mathrm{t}, 4 \mathrm{H})$, $6.78(\mathrm{~d}, 4 \mathrm{H}), 6.62(\mathrm{t}, 4 \mathrm{H}), 3.93(\mathrm{~m}, 8 \mathrm{H}), 1.79(\mathrm{~m}, 8 \mathrm{H}), 1.51$ $(\mathrm{m}, 8 \mathrm{H}), 0.23(\mathrm{~s}, 18 \mathrm{H}) .{ }^{13} \mathrm{C} \mathrm{NMR}\left(75 \mathrm{MHz}, \mathrm{CDCl}_{3}\right) \delta$ : $159.2,157.4,144.3,144.2,139.6,136.3,133.4,132.5$, $131.4,127.6,127.5,126.1,115.0,114.3,113.6,113.5$, $67.8,67.5,29.2,29.1,25.8,0.1$.

4.1.3 单体 1,2-双[4-(4-乙炔基苯基烷氧基)苯基]-1,2-二 苯基乙烯 $[1(m)]$ 的合成

以单体 1,2-双[4-(4-乙炔基苯基丁氧基)苯基]-1,2-二 苯基乙烯 $[1(4)]$ 的合成步骤为例, 在 $250 \mathrm{~mL}$ 装有搅拌子 的圆底烧瓶中加入 $4.27 \mathrm{~g}(5 \mathrm{mmol}) \mathbf{5}(4), 0.84 \mathrm{~g}$ (15 $\mathrm{mmol}) \mathrm{KOH}, 100 \mathrm{~mL} \mathrm{THF}$ 和 $200 \mathrm{~mL}$ 甲醇. 在室温下搅 拌 $3 \mathrm{~h}$ 后, 浓缩溶液并将其倒入 $500 \mathrm{~mL}$ 盐酸溶液中(1 $\mathrm{mol} / \mathrm{L})$. 混合物用二氯甲烷萃取四次. 收集有机相, 用 无水 $\mathrm{MgSO}_{4}$ 干燥, 旋蒸浓缩. 所得粗产物过硅胶柱[淋 洗液: $V($ 氯仿 $): V($ 正己烷 $)=1: 2]$ 纯化, 得到浅黄色固 体产物, 产率为 93.2\%. IR (film) $v$ : 3284, 3038, 2954, 2928, 2870, 2106, 1876, 1604, 1570, 1506, 1470, 1442, 1386, 1286, 1240, 1170, 1108, $1048 \mathrm{~cm}^{-1}$. ${ }^{1} \mathrm{H}$ NMR $(300$ $\left.\mathrm{MHz}, \mathrm{CDCl}_{3}\right) \delta: 7.43(\mathrm{~d}, 4 \mathrm{H}), 7.08(\mathrm{~m}, 10 \mathrm{H}), 6.91(\mathrm{~m}, 4 \mathrm{H})$, $6.83(\mathrm{~d}, 4 \mathrm{H}), 6.62(\mathrm{t}, 4 \mathrm{H}), 3.96(\mathrm{~m}, 8 \mathrm{H}), 2.99(\mathrm{~s}, 2 \mathrm{H}), 1.93$ $(\mathrm{m}, 8 \mathrm{H}) .{ }^{13} \mathrm{C}$ NMR $\left(75 \mathrm{MHz}, \mathrm{CDCl}_{3}\right) \delta: 159.6,158.0$, $145.2,144.6,140.1,137.0,134.3,133.2,132.1,128.3$, $128.2,126.8,115.1,114.2,114.1,93.5,76.4,68.2,67.8$,
26.6. HRMS (MALDI-TOF): calcd for $\mathrm{C}_{50} \mathrm{H}_{44} \mathrm{O}_{4}$, 708.3240, found 708.3303. 单体 1,2-双[4-(4-乙炔基苯基 己氧基)苯基]-1,2-二苯基乙烯[1(6)]以 $4.55 \mathrm{~g}$ (5 mmol) 5(6)为原料通过与 1(4)类似的合成步骤制得，为浅黄色 固体, 产率 95.6\%. IR (film) v: 3284, 3044, 2940, 2864, 2106, 1604, 1570, 1506, 1472, 1388, 1288, 1242, 1172, 1110, 1070, $1026 \mathrm{~cm}^{-1} .{ }^{1} \mathrm{H}$ NMR $\left(300 \mathrm{MHz}, \mathrm{CDCl}_{3}\right) \delta$ : $7.42(\mathrm{~d}, J=9.0 \mathrm{~Hz}, 4 \mathrm{H}), 7.11 \sim 7.00(\mathrm{~m}, 10 \mathrm{H}), 6.95 \sim 6.87$ $(\mathrm{m}, 4 \mathrm{H}), 6.83(\mathrm{~d}, J=9.0 \mathrm{~Hz}, 4 \mathrm{H}), 6.62(\mathrm{t}, J=9.0 \mathrm{~Hz}, 4 \mathrm{H})$, $3.98 \sim 3.86(\mathrm{~m}, 8 \mathrm{H}), 2.99(\mathrm{~s}, 2 \mathrm{H}), 1.80 \sim 1.77(\mathrm{~m}, 8 \mathrm{H})$, $1.50 \sim 1.39(\mathrm{~m}, 8 \mathrm{H}) .{ }^{13} \mathrm{C}$ NMR $\left(75 \mathrm{MHz}, \mathrm{CDCl}_{3}\right) \delta: 159.4$, $157.4,144.3,144.2,139.6,138.1,136.3,136.2,133.5$, $132.5,131.4,127.6,127.5,126.1,116.9,114.4,113.9$, 113.6, 113.5, 83.7, 75.7, 67.8, 67.5, 29.2, 29.1, 25.8. HRMS (MALDI-TOF): calcd for $\mathrm{C}_{54} \mathrm{H}_{52} \mathrm{O}_{4}, 764.3866$, found 764.3868 .

4.1 .4 单体 1,2-双[4-(炔丙基羟苯基)苯基]-1,2-二苯基乙 烯(2)的合成

在 $250 \mathrm{~mL}$ 圆底烧瓶中加入 $3.6 \mathrm{~g}$ (10 mmol) 1,2-双 (4-着基苯基)-1,2-二苯基乙烯(6), $3.57 \mathrm{~g}$ (30 mmol)炔丙 基溴, $6.91 \mathrm{~g}(50 \mathrm{mmol}) \mathrm{K}_{2} \mathrm{CO}_{3}$ 以及 $150 \mathrm{~mL}$ 丙酮. 混合物 加热回流，过夜反应。反应结束后，过滤，旋转浓缩滤 液. 粗产物经硅胶柱纯化 [淋洗液: $V($ 氯仿 $): V$ (正己烷) $=1: 2]$, 得到白色固体产物, 产率为 $83.2 \%$. IR (film) $v$ : 3288, 3050, 3024m, 2956, 2922, 2860, 2126, 1892, 1604, $1574,1506,1442,1370,1286,1236,1220,1176,1112$, 1974, $1024 \mathrm{~cm}^{-1} .{ }^{1} \mathrm{H}$ NMR $\left(300 \mathrm{MHz}, \mathrm{CDCl}_{3}\right) \delta: 7.10(\mathrm{~m}$, $6 \mathrm{H}), 7.04(\mathrm{~m}, 4 \mathrm{H}), 6.96(\mathrm{~m}, 4 \mathrm{H}), 6.70(\mathrm{t}, 4 \mathrm{H}), 4.63(\mathrm{t}, 4 \mathrm{H})$, 2.50 (s, 2H). ${ }^{13} \mathrm{C}$ NMR (75 MHz, $\left.\mathrm{CDCl}_{3}\right) \delta: 156.0,144.0$, $139.7,137.2,132.5,131.3,127.7,127.6,126.3,114.0$, 113.9, 78.6, 75.4, 55.8. HRMS (MALDI-TOF): calcd for $\mathrm{C}_{32} \mathrm{H}_{24} \mathrm{O}_{2}, 440.1776$, found 440.1778 .

\section{2 偶联聚合反应}

Glaser-Hay 偶联反应在 $\mathrm{CuCl}$ 和 TMEDA 在 $o$-DCB 的混合物中进行, 具体的操作步骤以 1(4)单体的聚合反 应为例, 在装有搅拌子的 $20 \mathrm{~mL}$ 试管中加入 $2 \mathrm{mg}(0.02$ mmol) $\mathrm{CuCl}, 8 \mathrm{mg}(0.07 \mathrm{mmol})$ TMEDA 以及 $4 \mathrm{~mL}$ $o$-DCB. 催化剂混合物在用压缩空气缓慢鼓气的条件下 在 $60{ }^{\circ} \mathrm{C}$ 的油浴中加热 $10 \mathrm{~min}$. 二炔烃单体 1(4) (0.3 g, $0.4 \mathrm{mmol}$ )溶在 $4 \mathrm{~mL} o$-DCB 后加入到催化剂混合物中. 搅拌 $3 \mathrm{~h}$ 后, 将反应混合物倒入到 $500 \mathrm{~mL}$ 经 $1 \mathrm{~mL} \mathrm{HCl}$ $(w=37 \%)$ 溶液酸化的甲醇中, 终止聚合反应. 沉淀静置 过夜，之后过滤、用甲醇洗涤，室温下真空干燥至恒重. 所得各聚合产物的表征数据如下:

P1(4): 白色粉末; 产率 $92.9 \% . M_{\mathrm{w}} 18300 ; M_{\mathrm{w}} / M_{\mathrm{n}} 1.9$ (GPC，聚苯乙烯校准). IR (film) v: 3048，2946，2870, 2136, 1884, 1602, 1566, 1504, 1470, 1442, 1384, 1288, 1242, 1168, 1108, 1046, $1015 \mathrm{~cm}^{-1}$. ${ }^{1} \mathrm{H}$ NMR (300 MHz, 
$\left.\mathrm{CDCl}_{3}\right) \delta: 7.43,7.24,7.09,7.05,7.02,6.92,6.81,6.62$, 3.97, 1.92. ${ }^{13} \mathrm{C}$ NMR (75 MHz, $\left.\mathrm{CDCl}_{3}\right) \delta: 159.6,157.3$, $144.3,144.2,139.6,136.4,134.1,134.0,132.5,131.4$, $127.6,126.2,114.6,114.1,113.8,113.6,113.5,81.3,73.0$, $67.6,67.1,25.9$.

P1(6): 白色粉末; 产率 $86.7 \% . M_{\mathrm{w}} 14800 ; M_{\mathrm{w}} / M_{\mathrm{n}} 1.7$ (GPC，聚苯乙烯校准). IR (film) v: 3042，2936，2860, 2136, 1882, 1602, 1564, 1504, 1468, 1384, 1286, 1244, 1168, 1108, $1012 \mathrm{~cm}^{-1} .{ }^{1} \mathrm{H}$ NMR $\left(300 \mathrm{MHz}, \mathrm{CDCl}_{3}\right) \delta$ : $7.44,7.07,6.92,6.83,6.62,3.96,3.85,2.06,1.51 .{ }^{13} \mathrm{C}$ NMR (75 MHz, $\left.\mathrm{CDCl}_{3}\right) \delta: 159.7,157.4,144.3,144.2$, $139.6,138.2,138.1,136.3,136.2,134.0,133.9,132.5$, $131.4,127.5,126.1,116.9,114.6,113.7,113.6,113.5$, 82.4, 81.3, 72.9, 67.9, 67.5, 29.2, 29.0, 25.8.

P2: 白色粉末; 产率 $71.9 \% . M_{\mathrm{w}} 17600 ; M_{\mathrm{w}} / M_{\mathrm{n}} 2.7$ (GPC, 聚苯乙烯校准). IR (film) v: 3052, 3030, 2904, 2852, 1882, 1604, 1574, 1506, 1442, 1362, 1288, 1212, 1176, 1110, 1074, $1026 \mathrm{~cm}^{-1} .{ }^{1} \mathrm{H}$ NMR (300 MHz, $\left.\mathrm{CDCl}_{3}\right)$ $\delta: 7.18,6.92,6.63,4.65 .{ }^{13} \mathrm{C}$ NMR $\left(75 \mathrm{MHz}, \mathrm{CDCl}_{3}\right) \delta$ : $155.8,143.9,139.7,137.3,137.1,132.5,131.4,127.7$, 127.6, 126.3, 113.9, 74.5, 70.9, 56.1.

\section{3 光刻蚀图案化}

聚合物薄膜的光交联反应在室温空气环境中在波 长为 $365 \mathrm{~nm}$ 的 UV 光照射下进行. 光源为 Spectroline ENF-280C/F UV 灯, 光源与薄膜间距离 $1 \mathrm{~cm}$. 入射光强 度约为 $18.5 \mathrm{~mW} / \mathrm{cm}^{2}$. 薄膜通过旋涂聚合物的 1,2 -二氯 乙烷溶液( $10 \% \mathrm{w} / \mathrm{w}$ ) 在硅片上制得(转速 $2000 \mathrm{r} / \mathrm{min}$, 时 长 $1 \mathrm{~min}$ ). 所得聚合物薄膜在室温下真空干燥过夜. 将 该聚合物薄膜在空气中曝露于通过铜制光掩膜的 UV 光 下照射 $5 \mathrm{~min}$, 产生光刻胶图案, 之后使用光学显微镜 (Nikon 80i)采用一般室内光源和 UV 光源条件拍照.

\section{References}

[1] (a) Cheuk, K. K. L.; Li, B. S.; Tang, B. Z. In Encyclopedia of Nanoscience and Nanotechnology, Vol. 8, Ed.: Nalwa, H. S., American Scientific Publishers, California, 2004, pp. 703 713; (b) Lam, J. W. Y.; Tang, B. Z. J. Polym. Sci. Part A: Polym. Chem. 2003, 41, 2607; (c) Cheuk, K. K. L.; Li, B. S.; Tang, B. Z. Curr. Trends Polym. Sci. 2002, 7, 41. (d) Tang, B. Z. Polym. News 2001, 26, 262; (e) Li, Z.; Dong, Y. Q.; Qin, A. J.; Lam, J. W. Y.; Dong, Y. P.; Yuan, W. Z.; Sun, J. Z.; Hua, J. L.; Wong, K. S.; Tang, B. Z. Macromolecules 2006, 39, 467.

[2] (a) Liu, J. Z.; Lam, J. W. Y.; Tang, B. Z. Chem. Rev. 2009, 109,
5799; (b) Zhou, L.; Jiang, H. F.; Huang, J. M.; Tang, J. Y. Chinese J. Org. Chem. 2006, 26, 1. (周否, 江焕峰, 黄精美, 唐金玉, 有机化 学, 2006, 26, 1.); (c) Häußler, M.; Lam, J. W. Y.; Qin, A.; Tse, K. K. C.; Li, M. K. S.; Liu, J.; Jim, C. K. W.; Gao, P.; Tang, B. Z. Chem. Commun. 2007, 2584; (d) Qin, A.; Lam, J. W. Y.; Dong, H.; Lu, W.; Jim, C. K. W.; Dong, Y. Q.; Häußler, M.; Sung, H. H. Y.; Williams, I. D.; Wong, G. K. L.; Tang, B. Z. Macromolecules 2007, 40, 4879; (e) Dong, H.; Zheng, R.; Lam, J. W. Y.; Häußler, M.; Tang, B. Z. Macromolecules 2005, 38, 6382; (f) Jim, C. K. W.; Qin, A.; Lam, J. W. Y.; Häußler, M.; Liu, J.; Yuen, M. M. F.; Kim, J. K.; Ng, K. A.; Tang, B. Z. Macromolecules 2009, 42, 4099.

[3] Jakubiak, R.; Collison, C. J.; Wan, W. C.; Rothberg, L. J.; Hsieh, B. R. J. Phys. Chem. A 1999, 103, 2394.

[4] Luo, J.; Xie, Z.; Lam, J. W. Y.; Cheng, L.; Chen, H.; Qiu, C.; Kwok, H. S.; Zhao, X.; Liu, Q.; Zhu, D.; Tang, B. Z. Chem. Commun. 2001, 1740 .

[5] (a) Qian, L. J.; Tong, B.; Zhi, J. G.; Yang, F.; Shen, J. B.; Shi, J. B.; Dong, Y. P. Acta Chim. Sinica 2008, 66, 1134. (钱立军，佟斌，支俊 格, 杨帆, 申进波, 石建兵, 董宇平, 化学学报, 2008, 66, 1134.); (b) Xun, Z. Q.; Tang, H. Y.; Zeng, Y.; Chen, J. P.; Yu, T. J.; Zhang, X. H.; Li, Y. Acta Chim. Sinica 2015, 73, 819. (寻知庆，唐海云，曾 毅, 陈金平，于天君，张小辉，李姫，化学学报， 2015，73，819.); (c) Mei, J.; Leung, N. L. C.; Kwok, R. T. K.; Lam, J. W. Y; Tang, B. Z. Chem. Rev. 2015, 115, 11718; (d) Zhang, Y. H.; Kong, L. W.; Shi, J. B.; Tong, B.; Zhi, J. G.; Feng, X.; Dong, Y. P. Chin. J. Chem. 2015, 33, 701.

[6] (a) Hu, R. R.; Leung, N. L. C.; Tang, B. Z. Chem. Soc. Rev. 2014, 43, 4494; (b) Wu, W. B.; Tang, R. L.; Li, Q. Q.; Li, Z. Chem. Soc. Rev. 2015, 44, 3997; (c) Hu, R. R.; Li, W. Z.; Tang, B. Z. Macromol. Chem. Phys. 2016, 217, 213.

[7] (a) Qin, A.; Lam, J. W. Y.; Tang, L.; Jim, C. K. W.; Zhao, H.; Sun, J.; Tang, B. Z. Macromolecules 2009, 42, 1421; (b) Qin, A.; Tang, L.; Lam, J. W. Y.; Jim, C. K. W.; Yu, Y.; Zhao, H.; Sun, J.; Tang, B. Z. Adv. Funct. Mater. 2009, 19, 1891.

[8] (a) Ogawa, T. Prog. Polym. Sci. 1995, 20, 943; (b) Hay, A. S. J. Polym. Sci. Part A: Polym. Chem. 1998, 36, 505; (c) Siemsen, P.; Livingston, R. C.; Diederich, F. Angew. Chem. Int. Ed. 2000, 39, 2633; (d) Bunz, U. H. F. Chem. Rev. 2000, 100, 1605.

[9] (a) Higashihara, T.; Ueda, M. Macromolecules 2015, 48, 1915; (b) You, N.-H.; Suzuki, Y.; Higashihara, T.; Ando, S.; Ueda, M. Polymer 2009, 50, 789 .

[10] (a) Häußler, M.; Lam, J. W. Y.; Qin, A.; Tse, K. K. C.; Li, M. K. S.; Liu, J.; Jim, C. K. W.; Gao, P.; Tang, B. Z. Chem. Commun. 2007, 2584; (b) Liu, J.-G.; Ueda, M. J. Mater. Chem. 2009, 19, 8907.

[11] (a) Raman, C. V. Proc. Ind. Acad. Sci. 1950, 31A, 141; (b) Inoue, T.; Okamoto, H.; Osaki, K. Macromolecules 1991, 24, 5670; (c) Inoue, T.; Hwang, E.-J.; Osaki, K. Polymer 1997, 38, 1029.

[12] Wei, Q.; Pötzsch, R.; Liu, X. L.; Komber, H.; Kiriy, A.; Voit, B. Will, P.-A.; Lenk, S.; Reineke, S. Adv. Funct. Mater. 2016, 26, 2545.

[13] (a) Yang, C. J.; Jenekhe, S. A. Chem. Mater. 1995, 7, 1276; (b) Yang, C. J.; Jenekhe, S. A. Chem. Mater. 1994, 6, 196; (c) Liu, Y. J.; Lam, J. W. Y.; Tang, B. Z. Natl. Sci. Rev. 2015, 2, 493; (d) Han, T.; Deng, H. Q.; Yu, C. Y. Y.; Gui, C.; Song, Z. G.; Kowk, R. T. K.; Lam, J. W. Y.; Tang, B. Z. Polym. Chem. 2016, 7, 2501.

[14] (a) Chu, J. B.; Huang, S. M.; Zhu, H. B.; Xu, X. B.; Sun, Z.; Chen, Y. W.; Huang, F. Q. J. Non-Cryst. Solids 2008, 354, 5480; (b) Chan, C. Y. K.; Lam, J. W. Y.; Jim, C. K. W.; Sung, H. H. Y.; Williams, I. D.; Tang, B. Z. Macromolecules 2013, 46, 9494.

[15] (a) Campbell, M.; Sharp, D. N.; Harrison, M. T.; Denning, R. G.; Turberfield, A. J. Nature 2000, 404, 53; (b) Li, Z. A.; Jiang, Z. Q.; Ye, S. H.; Jim, C. K. W.; Yu, G.; Liu, Y. Q.; Qin, J. G.; Tang, B. Z.; Li, Z. J. Mater. Chem. 2011, 21, 14663. 